\title{
Atomic-Nuclear Coupling Experiments
}

J. A. Becker

November 8, 2005

12th Conference on Capture Gamma-ray Spectroscopy and Related Topics South Bend, IN, United States September 4, 2005 through September 9, 2005 
This document was prepared as an account of work sponsored by an agency of the United States Government. Neither the United States Government nor the University of California nor any of their employees, makes any warranty, express or implied, or assumes any legal liability or responsibility for the accuracy, completeness, or usefulness of any information, apparatus, product, or process disclosed, or represents that its use would not infringe privately owned rights. Reference herein to any specific commercial product, process, or service by trade name, trademark, manufacturer, or otherwise, does not necessarily constitute or imply its endorsement, recommendation, or favoring by the United States Government or the University of California. The views and opinions of authors expressed herein do not necessarily state or reflect those of the United States Government or the University of California, and shall not be used for advertising or product endorsement purposes. 


\title{
Atomic-Nuclear Coupling Experiments
}

\author{
J. A. Becker \\ Lawrence Livermore National Laboratory \\ Livermore, CA 94550
}

\begin{abstract}
Atomic-nuclear coupling experiments are described, with an emphasis on recent experiments aimed at demonstrating the NEET mechanism in atomic nuclei. Upper limits for $\mathrm{x}$-ray induced decay of the Hf-178 31-y isomer reported by Ahmad and his colleagues are presented, and these upper limits are contrasted with the positive reports of Collins and coworkers.
\end{abstract}

Keywords: atomic nuclear coupling, NEET, ${ }^{235} \mathrm{U}{ }^{189} \mathrm{Os}$, isomers.

PACS: 23.20.-g, 23.90.+W, 25.20.Dc, 27.70. $+\mathrm{q}$

\section{INTRODUCTION}

Manipulating a nucleus with atomic probes and potentially gaining nuclear energy release at the cost of atomic energy is an attractive idea. Basic science interests include identification of the mechanisms and respective cross sections, and the prospect of "energy on demand" has a strong applied science interest. Familiar isotopes discussed in this context are illustrated in Figure 1. Interest in atomic-nuclear coupling has been renewed with recent experiments and theoretical work focused on induced decay of the $31-\mathrm{y}$ isomer in ${ }^{178} \mathrm{Hf}$ at $\mathrm{E}_{\mathrm{x}}=2.4 \mathrm{MeV}$, initiated by $\mathrm{x}$-ray irradiation, and by experiments done with the goal of observing "Nuclear Excitation by Electronic Transition" (NEET). The NEET process has been observed successfully in x-ray irradiation of ${ }^{197} \mathrm{Au}$, while only cross-section limits have been obtained for ${ }^{187} \mathrm{Os}$. Attempts have been made to excite the first excited state of ${ }^{235} \mathrm{U}$ and $77 \mathrm{eV}\left(t_{1 / 2} \sim 25 \mathrm{~m}\right)$ in a laser-induced plasma. These experiments together with theoretical calculations are discussed in the next sections. A "Workshop on Nuclear Isomers" organized by Prof. Yang Sun preceded CGS12. This workshop topics featured aspects of the many roles isomer studies have played in nuclear physics. Olivier Roig presented a poster at CGS12 describing superelastic scattering work, reporting an indirect measure of superelastic scattering for isomeric ${ }^{177} \mathrm{Lu}-200 \mathrm{~b}$. 

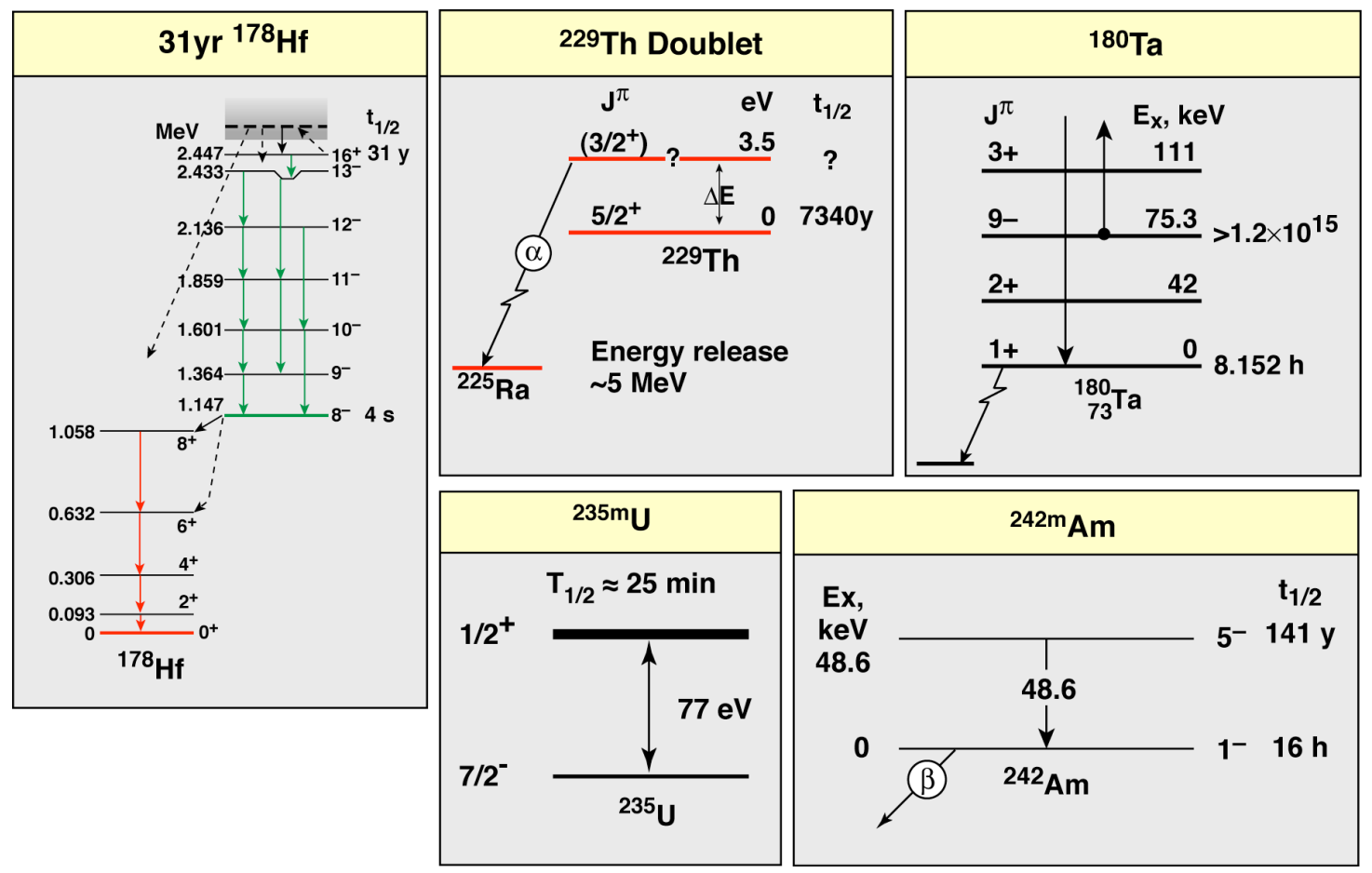

FIGURE 1. Nuclear isomers often mentioned in connection with atomic-nuclear coupling.

\section{X-Ray Induced Decay of Nuclear Isomers}

The 31-y isomer in ${ }^{178} \mathrm{Hf}\left({ }^{178 m 2} \mathrm{Hf}\right)$ has attracted considerable interest, because of the potential high energy release and the isomer's long half life. ${ }^{178 m 2} \mathrm{Hf}$ is available in research quantities mixed with ${ }^{178} \mathrm{Hf}$, and often other $\mathrm{Hf}$ isotopes, depending on the method of production. The positive reports of induced decay by Collins, et. al., ${ }^{1}$ (Citation is to the most recent work, where citations to earlier work may be found.) was quickly criticized as improbable because of the overwhelming E1 strength required by the reported cross section, coupled with the requirement of a nearby intermediate state with special properties. Verification experiments by Ahmad, el. al., ${ }^{2}$ and Roberts, Carroll, et. al., ${ }^{3}$ set upper limits for enhanced decay of the ${ }^{178 m 2} \mathrm{Hf}$ many orders magnitude below the continued positive reports of Collins and his coworkers. Results are compared in Figure 2. Belief in X-ray induced enhanced decay of the 2.4$\mathrm{MeV}$ isomer with any practical cross section is contrary to scientific evidence. The discussion continues in popular literature. ${ }^{4}$ Suggestions abound for practical applications. ${ }^{5}$ Numerous popular press articles also are in evidence, and the discussion has spread abroad. 


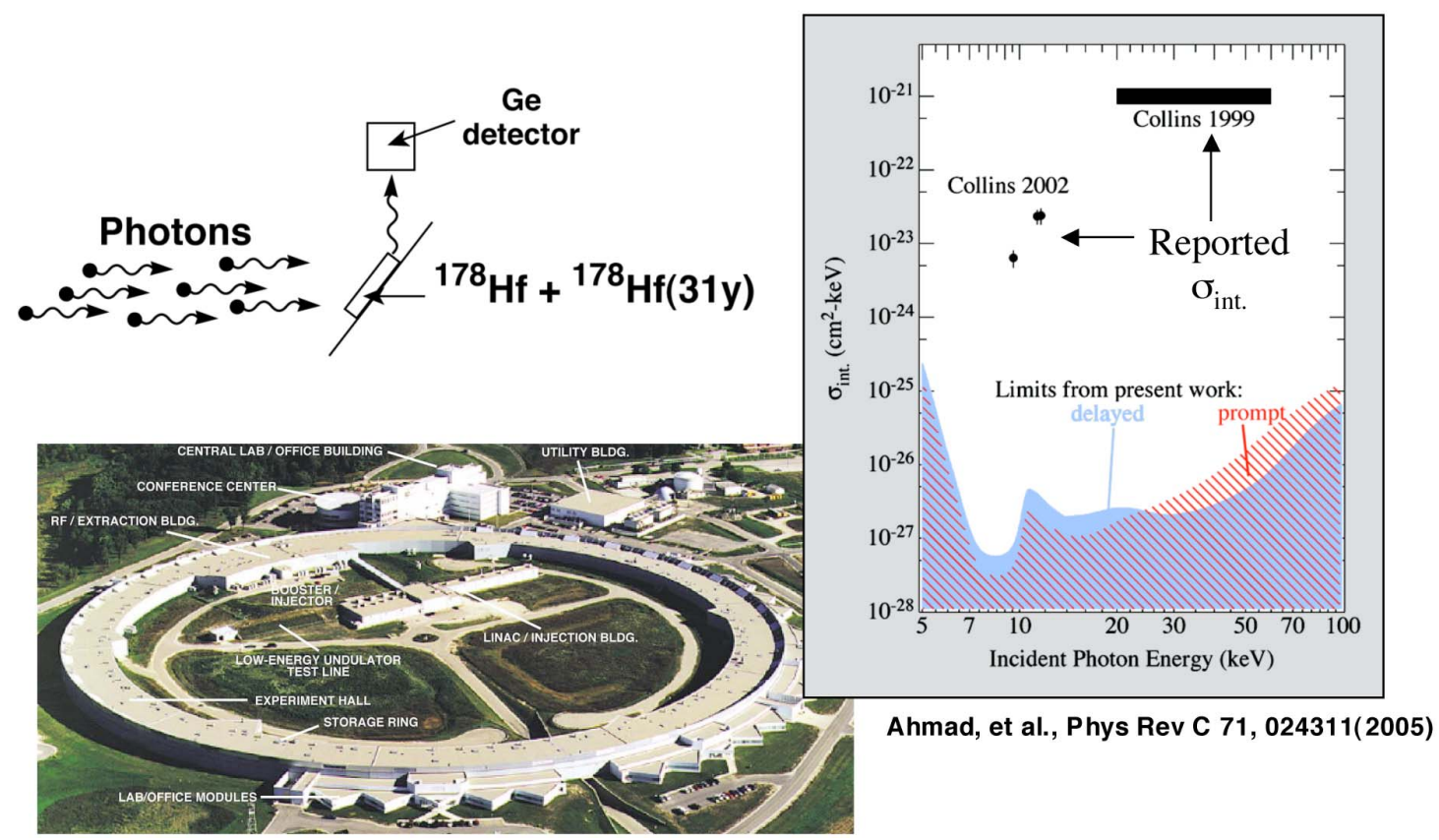

FIGURE 2. A cartoon of the experimental arrangement employed by Ahmad, et. al., ${ }^{2}$ upper limits to enhanced decay of isomeric ${ }^{178} \mathrm{Hf}$ obtained in their work, and a visual of the Advanced Photon Source. The upper limits come in 2 pieces: for enhanced decay through the $8^{-}$isomeric state in ${ }^{178} \mathrm{Hf}$, and for decay which bypasses that state. Ahmad, et al., used a "white" beam of incident x-rays. Roberts, Carroll et. al., ${ }^{3}$ have reported a cross sections limit of $10^{-25} \mathrm{keV}-\mathrm{cm}^{2}$, covering (stepwise) $75 \%$ of a narrow region of incident $\mathrm{x}$-ray energies $9.45-13.5 \mathrm{keV}$ (not illustrated).

\section{NEET}

Morita $^{6}$ pointed to the possibility of NEET, suggesting NEET as a possibility of ${ }^{235} \mathrm{U}$ and ${ }^{235 m} \mathrm{U}$ separation. Kishimoto ${ }^{7}$ presented evidence for NEET in ${ }^{197} \mathrm{Au}$, obtained in a daunting experiment at SP-ring8, The probability of NEET, reporting for the particular atomic excitation is $\mathrm{P}(\mathrm{NEET})=5.0 \pm 0.6 \times 10^{-8} / \mathrm{k}$-vacancy, is good agreement with calculation $\sim 4 \times 10^{-8} / \mathrm{k}$-vacancy. ${ }^{8,9}$ Configurations are given in Figure 3. Upper limits to the NEET cross section for ${ }^{189} \mathrm{Os}$ for populating the $69.537 \mathrm{keV} \mathrm{5/2}$ excited state in ${ }^{189}$ Os are reported by Ahmad, et. al., ${ }^{10}$ and by Akoi, et. al., ${ }^{11}$ as $\mathrm{P}(\mathrm{NEET})<10^{-10} / \mathrm{k}$-vacancy, again in agreement with calculations of Tkalya and Harston. See Figure 4. 

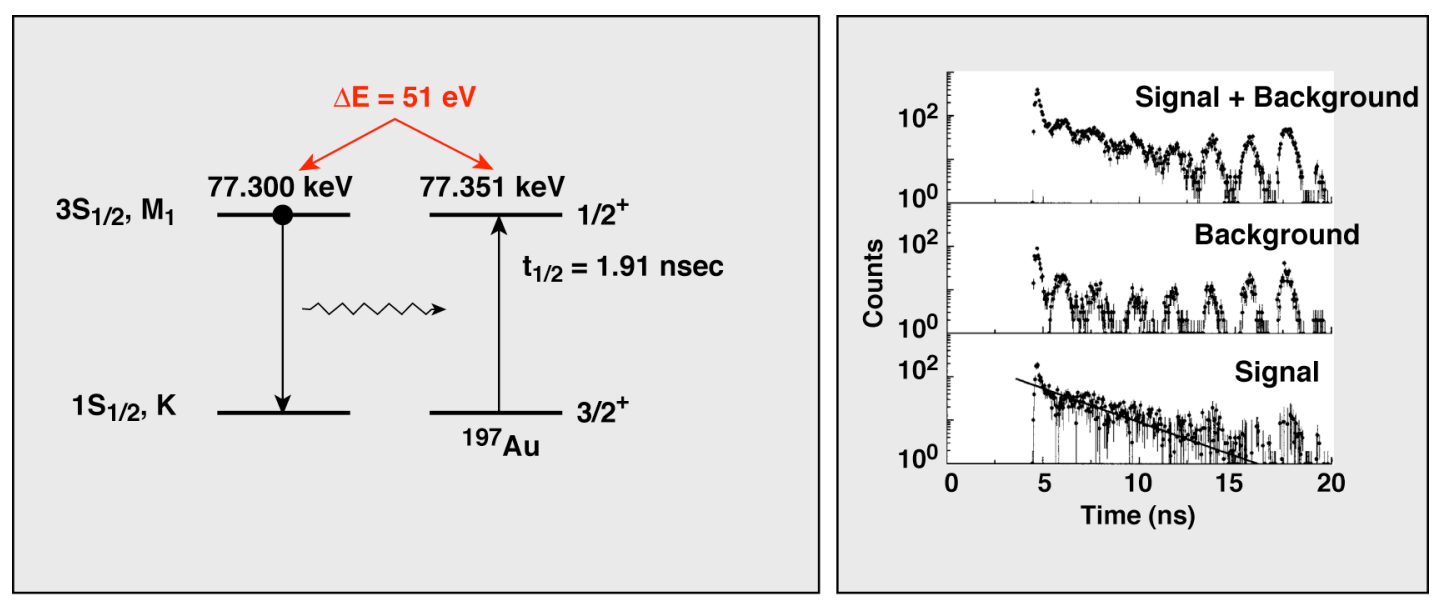

\begin{tabular}{|c|c|}
\hline Experiment & Theory \\
\hline $\mathrm{P}_{\mathrm{NEET}}=5.0 \pm 0.6 \times 10^{-8} / \mathrm{k}$ vacancy $(\mathrm{a})$ & $\mathrm{P}_{\mathrm{NEET}}=3.8 \times 10^{-8} / \mathrm{k}$ vacancy $(\mathrm{b})$ \\
& $\mathrm{P}_{\mathrm{NEET}}=3.8 \times 10^{-8} / \mathrm{k}$ vacancy $(\mathrm{c})$ \\
\hline
\end{tabular}

FIGURE 3. The relevant configuration in ${ }^{197} \mathrm{Au}$ for the NEET process, and the NEET signal observed by Kishimoto, et. al. ${ }^{7}$

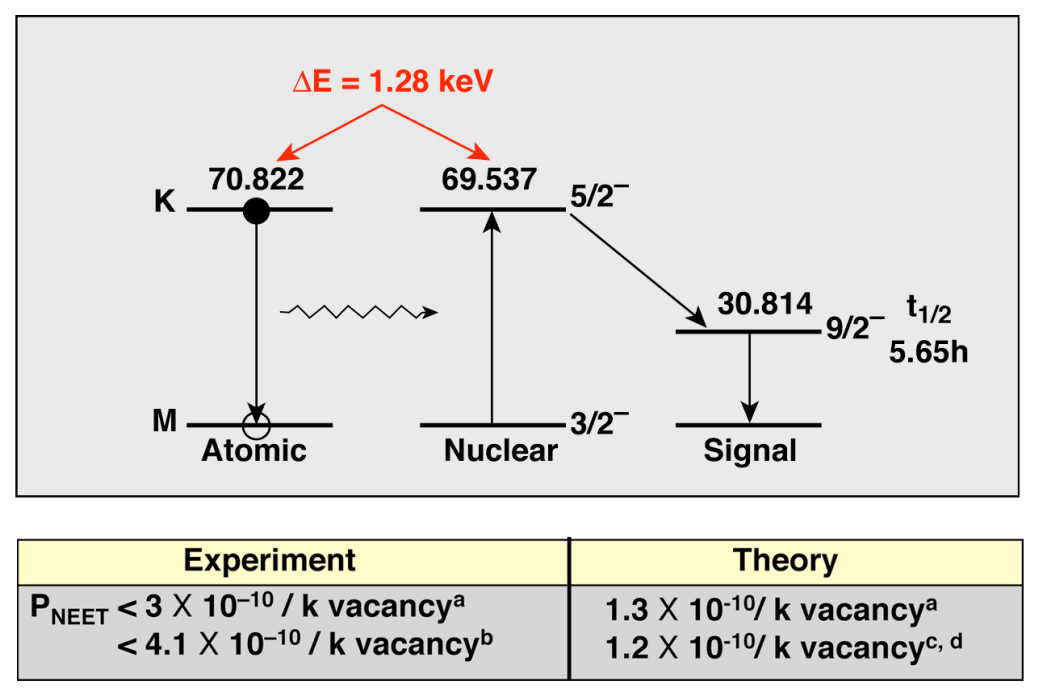

FIGURE 4. The relevant configurations in ${ }^{189}$ Os for the NEET process, and the upper limits obtained in the experiments of (a) Ahmad, et. al., ${ }^{10}$ and (b) Akoi, et. al., ${ }^{11}$ and the calculations of (c) Tkalya ${ }^{8}$ and (d) Harston ${ }^{9}$ for $\mathrm{P}_{\text {NEET }}$.

\section{NEET in $\mu$-Mesic Atoms}

NEET is observed in $\mu$-mesic atom studies. Here, because of the very high muonic atomic energies, the many continuum states enable the NEET condition of energy degeneracy and multiple matching. See, e.g., Bernow, et. al. ${ }^{12}$ and Engfer ${ }^{13}$. The 
emphasis was on measurements of nuclear quadrupole moments, using NEET as a population mechanism.

\section{NEET in a Plasma}

Meot and his collaborators have attempted to populate ${ }^{235 m} \mathrm{U}$ in a laser-induced plasma. ${ }^{14}$ Elaborate calculations are required in order to set appropriate laser conditions so that the desired plasma is obtained. Morel, et. al., ${ }^{15}$ have shown that the laser conditions required for an observation is more than present day lasers can deliver. Meot and his collaborators are turning away from ${ }^{235 m} \mathrm{U}$, and they are planning an experiment in ${ }^{201} \mathrm{Hg}$, ${ }^{16}$ where calculations show the chances of success are higher. The laser pulse required is $1 \mathrm{~ns}$ long, and 100 Joules.

\section{NEEC}

Meot and his collaborators ${ }^{16}$ are also mounting an experiment to search for Nuclear Excitation by Electron Conversion (NEEC) in ${ }^{57} \mathrm{Fe}$, taking advantage of inverse kinetics and the energetic beams available at GANIL.

\section{Dicke Radiation}

Robert Dicke first used the term superradiance in $1954^{17}$ to describe the emission of photons from a quantum state consisting of an ensemble of excited atoms coupled only by the electromagnetic field of the photon. This state maximizes when the number of excited states equals the number of ground states in the ensemble. The superradiant state emits photons at a rate proportional to the number of atoms in the ensemble squared. High isomer enrichment is required to enter the superradiant state An effort at Los Alamos is underway to develop a coherent photon source based on a Mössbauer crystal, specifically a Mössbauer crystal highly enriched $(>10 \%)$ in ${ }^{93 m} \mathrm{~N}_{\mathrm{b}}{ }^{18}$ Fortunately, ${ }^{93 \mathrm{~m}} \mathrm{Nb}$ is an isomer that can be obtained in high purity. It is produced in 89 percent abundance from the decay of ${ }^{93} \mathrm{Mo}$ and is produced in 95 percent abundance from ${ }^{93} \mathrm{Zr}$ decay. A single Mössbauer crystal is needed to achieve nuclear superradiance. The Mössbauer effect is necessary to permit the nuclei to be resonant with the emitted gamma ray. A crystal lattice is needed so that the nuclei will "feel" the electromagnetic field of the photon, in phase. Thus the superradiant state exists only along Bragg angles. Isomer collection is an issue, and crystal growing is difficult. An efficient crystal growing process was developed, and $\mathrm{KNbF}_{6}$ crystals $2 \mathrm{~mm}$ in length have been grown. The attempt to grow $\mathrm{K}^{93 m} \mathrm{NbF}_{6}$ crystals has stalled. This is a very exciting, long range research project.

\section{ACKNOWLEDGMENTS}

This work was funded in part by the U.S Department of Energy, and performed under the auspices of the U.S. Department of Energy by the University of California, Lawrence Livermore National Laboratory, Contract No. W-7405-Eng-48 (LLNL). 


\section{REFERENCES*}

*Reference cited usually contains citations to the earlier work, which may be more appropriate in context.

1. C.B. Collins, et. al., Laser Physics 14, 154 (2004).

2. I. Ahmad, et. al., Phys. Rev. C 71, 024311 (2005).

3. J. J. Carroll, et. al., Laser Phys. Lett. 1,275 (2004).

4. B. Schwarzschild, Physics Today 57, 21 (May 2004); P. M. Walker and J. J. Carroll, Physics Today 58, 39 (June 2005).

5. D. Hambling, in NewScientist.com, 13 Aug. 2003.

6. M. Morita, Prog. Theor. Phys. 49, 1574 (1973).

7. S. Kishomoto, et. al., Phys. Rev. Lett. - 85, 1831 (2001).

8. E.V. Tkalya, in AIP Conference Proceedings 506, 486 (2000); E.V. Tkalya, Phys. Rev. C 71, 024606 (2005); E.V. Tkalya, Physics Uspekhi 48, 525 (2005).

9. M. Harston and J. J. Carroll, Laser Physics 15, 487 (2005).

10. I. Ahmad, et. al., Phys. Rev. C 61, R05304 (2000). See also the discussion by D. Gemmell, in AIP Conference Proceedings 652, 239 (2003).

11. K. Aoki, et. al., Hyperfine Int. 143, 111 (2002).

12. S. Bernow, et. al., Phys. Rev. Lett. 21, 457 (1968).

13. R. Engfer, in Proceedings of the International Conference on Nuclear Physics vol. 2, p. 437, Munich 1973, edited by J. de Boer and H.J. Mang, North Holland/Elsevier.

14. G. Claverie, et. al., Phys. Rev. C 70, 044303 (2004).

15. P. Morel, et. al., Phys. Rev. A 69, 0634214 (2004).

16. V. Meot, private communications (2005).

17. R. H. Dicke, Phys. Rev. 93(1) 99 (1954).

18. J. Wilhelmy, private communication (2005). 